\title{
Cost-effectiveness analysis of low versus high dose colistin in the treatment of multi-drug resistant pneumonia in Saudi Arabia
}

\author{
Abdul Karim Suleman Cara ${ }^{1} \cdot$ Syed Tabish Razi Zaidi ${ }^{2} \cdot$ Fatima Suleman $^{3}$
}

Received: 1 August 2017 / Accepted: 3 August 2018 / Published online: 16 August 2018

C) Springer Nature Switzerland AG 2018

\begin{abstract}
Background Gram negative pathogens are increasingly resistant to commonly used first line antibiotics and colistin is in most cases the only medicine available. There is very limited information available comparing the effectiveness and costs of low versus high dose colistin with studies showing efficacy with both doses and with variable levels of adverse effects. The absence of a definite dosing strategy makes a model to compare low dose and high dose colistin invaluable in making decisions regarding the appropriate use of colistin. Objective This study was designed to evaluate the cost effectiveness of low versus high dose colistin in the treatment of Pneumonia caused by colistin-only sensitive gram negative bacteria from the perspective of a tertiary care hospital in Saudi Arabia. Setting 300-bed tertiary care hospital in Saudi Arabia. Method A retrospective review was conducted to compare the costs and outcomes of treatment of pneumonia with low versus high dose colistin. The model followed an average patient from initiation of treatment until clinical cure or failure. Main outcome measures The main outcomes were cure, nephrotoxicity, total direct costs per episode, cost per additional cure and cost per nephrotoxicity avoided. Results There was no significant difference between high and low dose colistin with regards to clinical cure (30\% vs. $21 \% ; p=0.292)$. Significantly more patients experienced nephrotoxicity with high versus low dose colistin (30\% vs. $8 \%$; $p=0.004)$. With low dose colistin the incremental costs per nephrotoxicity avoided was SAR-3056.28. One-way sensitivity analyses did not change the overall results. Conclusion Low dose was not inferior to high dose colistin in terms of clinical cure and had a lower incidence of nephrotoxicity resulting in significant cost avoidance.
\end{abstract}

Keywords Colistin $\cdot$ Cost effectiveness $\cdot$ Efficacy $\cdot$ Gram negative $\cdot$ Multi drug resistant $\cdot$ Pneumonia $\cdot$ Saudi Arabia

\section{Impacts on Practice}

- A low dose colistin treatment of hospital-acquired pneumonia is as effective as a high-dose regimen.

- A low dose colistin treatment of hospital-acquired pneumonia causes less nephrotoxicity than a high dose colistine treatment, and therefore should be the preferred treatment option.

Abdul Karim Suleman Cara

akcaraza@yahoo.com

1 King Abdulaziz Hospital, Ministry of National Guard-Health Affairs, Post Office Box 2477, Al-Hasa 31982, Saudi Arabia

2 School of Healthcare, University of Leeds, Leeds LS29JT, United Kingdom

3 School of Health Sciences, University of KwaZulu-Natal, Westville Campus, Private Bag X54001, Durban 4000, South Africa

\section{Introduction}

Nosocomial Pneumonia (NP) is defined as pneumonia that occurs after healthcare contact. NP encompasses hospitalacquired pneumonia (HAP) and ventilator-associated pneumonia (VAP) [1, 2]. Rates of HAP due to multidrug-resistant gram-negative bacteria (MDR-GNB) have escalated in recent years and are a major concern [3]. Gram negative pathogens are increasingly resistant to commonly used first line antibiotics and often colistin is the only medicine available against MDR-GNB. The incidence of VAP in developing countries ranges from 16.7 to 73.4 per 1000 ventilator-days in adult ICUs $[4,5]$. The effect of MDR bacterial infections has been studies and demonstrates an increase in overall costs [6].

Various studies have reported on the effectiveness of high dose colistin (HDC) ranging from 5 to $10 \mathrm{mg} / \mathrm{kg} / \mathrm{day}$ and low dose colistin less than $5 \mathrm{mg} / \mathrm{kg} /$ day (LDC) [7, 8]. There are still no definite dosing recommendations and limited 
information is available about the comparative effectiveness of LDC versus HDC. Studies on the safety and efficacy of colistin abound both with HDC $[9,10]$ and LDC $[7,8,11]$. Studies have shown efficacy with both these doses with variable level of adverse effects, mainly nephrotoxicity which is one of the most commonly cited adverse effects of colistin treatment [12-15].

There is convincing evidence of greater incidence of nephrotoxicity using higher doses of colistin. Falagas et al. [14] reported nephrotoxicity that had a statistically significant dose dependent relationship. Nephrotoxicity was reported to occur in $43 \%$ of patients by Pogue et al. [16], with greater nephrotoxicity with doses between 3 and $4.9 \mathrm{mg} / \mathrm{kg} /$ day. Chan et al. [17] reported a much higher rate of colistin associated nephrotoxicity using doses between 2.5 and $5 \mathrm{mg} / \mathrm{kg} / \mathrm{day}$.

Given the reported efficacy of LDC in the treatment of MDR GNB $[7,8,11]$ and the financial burden that NP places on the healthcare systems worldwide, the purpose of this study was to construct an economic model that would allow the comparison of LDC and HDC in the treatment of pneumonia caused by colistin-only sensitive MDR GNB.

\section{Aim of the study}

The aim of this study was to determine cost effectiveness of low dose colistin (less than $2.5 \mathrm{mg} / \mathrm{kg}$ ) versus high dose colistin (greater than $2.5 \mathrm{mg} / \mathrm{kg}$ ) dosed every $12 \mathrm{~h}$ in the treatment of NP due to MDR-GNB using a decision analytic (DA) model.

\section{Ethics approval}

Full ethical approval for the study was obtained from King Abdullah International Medical Research Centre (HAS-16437780-10741) on 19 January 2016 and from the Humanities and Social Sciences Research Ethics Committee of the University of KwaZulu-Natal (HSS/0975/015M) on 29 January 2016.

Patient confidentiality was maintained at all times and no patient hospital numbers, names or date of birth/identification numbers were reported in the data sets.

\section{Methods}

\section{Study design}

The design of this study was a retrospective analysis conducted at a 300-bed hospital in Saudi Arabia comparing the treatment of NP with low dose colistin versus high dose colistin. The study population consisted of all patients treated with colistin for Nosocomial Pneumonia caused by
MDR-GNB at King Abdulaziz Hospital, AlHasa, Saudi Arabia from July 2011 to December 2014. Between July 2011 and December 2012 patients were treated with LDC due to concerns of nephrotoxicity. This practice changed from July 2013 when physicians preferred to use HDC. Patients in the low dose colistin group received less than $2.5 \mathrm{mg} /$ $\mathrm{kg}$ and the high dose colistin group received greater than $2.5 \mathrm{mg} / \mathrm{kg}$ administered every $12 \mathrm{~h}$. The commercial product used was Colomycin ${ }^{\circledR}$ Injection by TEVA UK containing $33 \mathrm{mg}$ colistin base per vial (30,000 international units per $\mathrm{mg}$ ). Costing data for attending physician and consultant visits were calculated after reviewing the patient charts and noting the number of visits. Nursing costs were calculated from hospital costing data which quantified the daily cost of nurses in ICU and general ward.

\section{Definitions}

Multidrug-resistant gram negative bacteria for the purposes of this study are defined as bacteria resistant to aminoglycosides, anti-pseudomonas penicillins, carbapenems, cephalosporins and fluoroquinolones [18].

Clinical cure was defined as resolution of signs and symptoms of infection including absence of fever for a minimum of $72 \mathrm{~h}$ and a white blood cell count below 12,000 cells/ $\mathrm{mm}^{3}$.

Nephrotoxicity was defined as an increase in serum creatinine by $26.52 \mu \mathrm{mol} / \mathrm{L}$ or more within $48 \mathrm{~h}$ or increase in serum creatinine to 1.5 times baseline or more within the last 7 days [19].

\section{Participants}

There were a total of 171 patients that received colistin during the study period. After applying the inclusion and exclusion criteria 96 were evaluated. This included 63 and 33 in the LDC and HDC groups, respectively.

\section{Inclusion criteria}

The following patients were included in the study:

1. Patient over 12 years old and receiving colistin for documented nosocomial pneumonia in ICU and non-ICU.

2. Patients must have received colistin for colistin-only sensitive MDR-GNB infection.

3. Patients must have received colistin for at least $72 \mathrm{~h}$.

4. If a patient received more than one course of colistin within a single admission, only the first course was included. 


\section{Exclusion criteria}

The following patients were excluded from the study:

1. Age less than 12 years old.

2. Patients treated only with inhaled colistin.

3. Patients who did not receive colistin for at least $72 \mathrm{~h}$.

4. Patients with moderate to severe renal impairment or received renal replacement therapy or dialysis prior to colistin therapy.

\section{Statistical analyses}

In this study, two-sided significance was used for statistical analyses. All $p$ values of 0.05 or less were considered statistically significant. Dichotomous data were compared using the Chi Square test. Normally distributed continuous data are expressed as mean and standard deviation and compared using the Student's $t$ test. Otherwise, values are presented as medians with ranges and compared using the Mann-Whitney U test. Quantitative variables were analyzed with the Chi square test or Fischer's exact test (two-tailed) when necessary. All data was entered in a database and SPSS 20.0 software package was used to analyze the data.

\section{Model outcomes and pharmacoeconomic analyses}

The data was modelled using a decision analysis, considering the occurrence of NP with MDR-GNB, the two treatment alternatives (LDC vs. HDC) and two outcomes (cure and no cure) as well as nephrotoxicity in each. Figure 1 shows the decision tree used for cost-effectiveness analysis. Clinical outcomes (cure rate and nephrotoxicity rate) and the cost outcomes (total cost per patient) were calculated. Effectiveness was measured as rate of clinical cure and nephrotoxicity

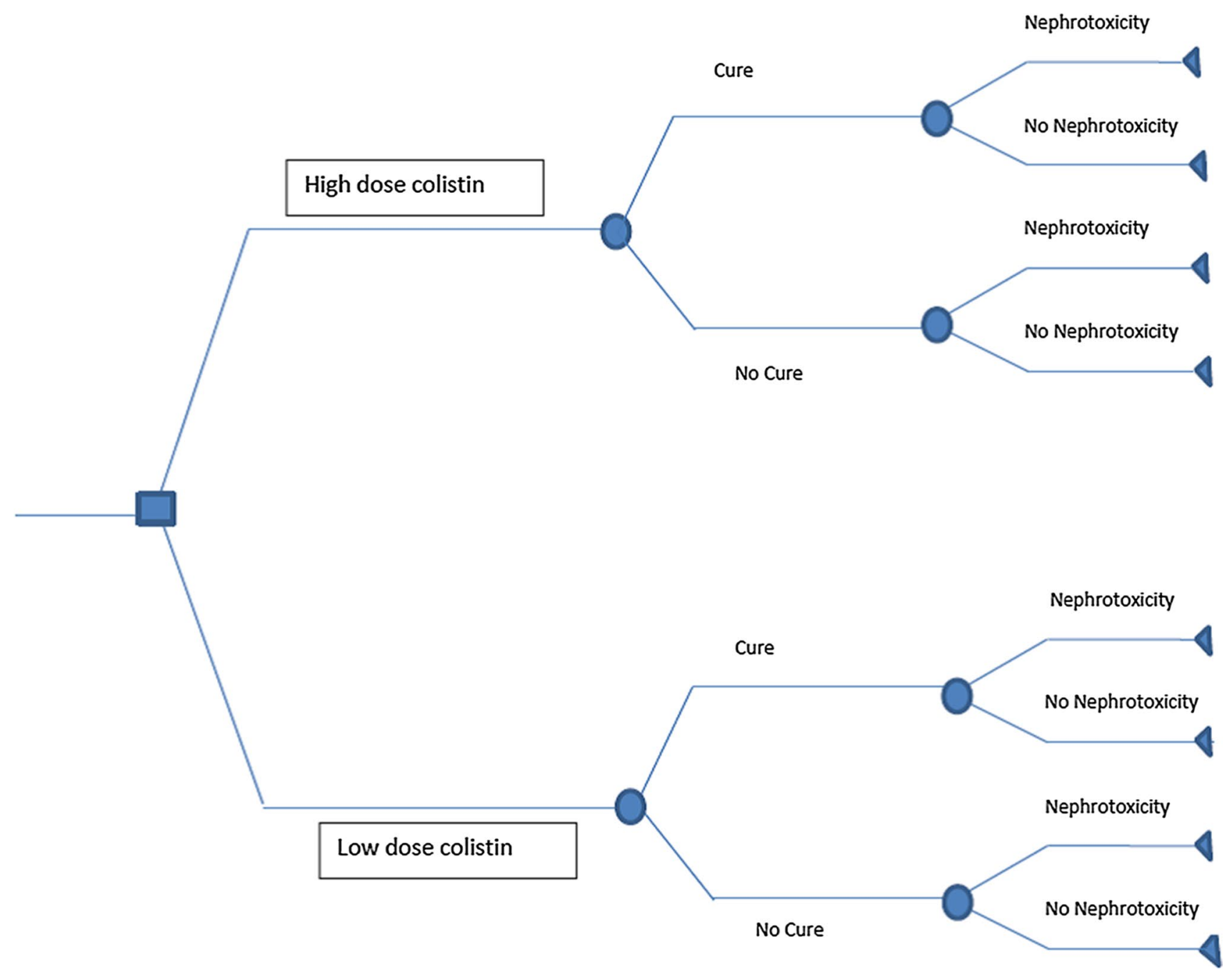

Fig. 1 Decision model used for cost-effectiveness analysis 
avoided. Probabilities of the different outcome parameters came from analysis of our retrospective study.

Incremental cost (IC) was calculated as Cost of HDC - Cost of LDC. Average cost effectiveness ratio (CER) was calculated as C LDCE LDC for the LDC strategy and C HDCE HDC for the HDC strategy; where $\mathrm{C}=$ cost (SAR = Saudi Arabian Riyal) and E=efficacy (cure or nephrotoxicity avoided). Efficacy was defined as the probability of patients who experienced NP responded to the study medicine (defined as 'cure') and probability that nephrotoxicity would be avoided in patient treated with the study medicine. Cost effectiveness was measured in terms of Incremental Cost Effectiveness Ratio (ICER).

ICER was calculated using the following equation:

(Cost of LDC) - (Cost of HDC) /(Efficacy of LDC)

- (Efficacy of HDC)

A dominant strategy was defined as being less costly with increased efficacy (clinical cure) or with least toxicity (nephrotoxicity).

\section{Costing}

Direct costs for each treatment option were calculated considering the ward costs, physician and consultant costs, nurse costs, laboratory costs and cost of colistin therapy. Direct non-medical costs such as transportation; indirect non-medical costs such as lost income; and intangible costs such as pain are not considered since they are beyond the scope of the study. Cost of consumables and administration sets as well as costs for pharmacy staff were not included as they were assumed to be identical for the two arms (prepared and administered in the same manner).

\section{Perspective and timescale}

This economic analysis was conducted from the perspective of the payer King Abdulaziz Hospital. The study period was that of the treatment period being from initiation of colistin to treatment cure or failure.

\section{Discounting and currency}

Future costs were not included since the costs and benefits pertained to the time horizon of colistin treatment. Discounting was therefore not performed because the time-period was too short to show any significant contribution. The currency used was SAR (USD 1 = SAR 3.77 as at 16 November 2016, www.xe.com).

\section{Assumptions}

Being a cost-effectiveness analysis, there were some assumptions made that could limit the generalizability of these results.

Parameters for adverse reactions (ADRs) other than nephrotoxicity were not included because they were assumed to have non-significant contributions to the overall costeffectiveness and because nephrotoxicity is the main adverse drug reaction affecting outcomes. The cost of antibiotics used to treat other gram-negative and gram-positive organisms were assumed to be equivalent between both groups and thus not included into the model. The costs of infection focused on NP due to Acinetobacter baumannii (AB), Pseudomonas aeruginosa (PA) and Klebsiella pneumonia (KP) and any treatment costs associated with other infections were not accounted for in the model.

\section{Results}

\section{Baseline characteristics}

The baseline characteristics of the samples are summarized in Table 1 . The two groups did not differ significantly with regards to age (68.76 years and 67.08 years; $p=0.707$ ), however, there was a significant difference with regards to weight $(56.52 \mathrm{~kg}$ and $76.87 \mathrm{~kg} ; p=<0.001)$ and gender (79\% and 56\% male; $p=0.025$ ) between the HDC and LDC groups, respectively.

Comorbidities between the two groups were not significantly different in all respects except for malignancy (2 vs. $0 ; p=0.048$ ) for the HDC and LDC groups, respectively. The Charlson comorbidity index between the two groups (3.88 and $3.67 ; p=0.585$ ) was not significantly different for the HDC and LDC groups, respectively.

There was no significant difference with respect to concomitant nephrotoxic medications (vancomycin, aminoglycosides, Non-Steroidal Anti-Inflammatory Drugs, Angiotensin Converting Enzyme Inhibitors, Angiotensin II receptor blockers and furosemide) used in the two groups. The average duration of colistin treatment, average length of stay and location of patients within the hospital between the two groups was not significantly different.

The two groups were significantly different in terms of the causative organism; $\mathrm{AB}(76 \%$ and $53 \% ; p=0.037)$, PA ( $24 \%$ and $49 \% ; p=0.018$ ), for HDC and LDC, respectively. However, KP (6\% and 9\%; $p=0.56)$ was not significantly different, for HDC and LDC, respectively. The average dose $(\mathrm{mg})$ of colistin was $2.83 \pm-0.50$ and $1.52 \pm 0.43$ $(p=0.001)$ and the average duration of colistin treatment (days) was $12.76 \pm 13.38$ and $12.67 \pm 12.51(p=0.974)$ in the HDC and LDC groups, respectively. 
Table 1 Characteristics of patients based on colistin dose group

\begin{tabular}{|c|c|c|c|}
\hline & $\begin{array}{l}\text { High dose colistin } \\
(\mathrm{n}=33) \mathrm{n}(\%)\end{array}$ & $\begin{array}{l}\text { Low dose colistin } \\
(\mathrm{n}=63) \mathrm{n}(\%)\end{array}$ & $p$ value \\
\hline \multicolumn{4}{|l|}{ Demographics } \\
\hline Age in years & $68.76 \pm 20.61$ & $67.08 \pm 20.77$ & 0.707 \\
\hline Weight in $\mathrm{kg}$ & $56.52 \pm 17.42$ & $76.870 \pm 24.87$ & 0.000 \\
\hline Gender (male) & $26(78.8)$ & $35(55.6)$ & 0.025 \\
\hline Gender (female) & $7(21.2)$ & $28(44.4)$ & 0.025 \\
\hline \multicolumn{4}{|l|}{ Comorbidities } \\
\hline Charleston comorbidity score & $3.88 \pm 1.71$ & $3.67 \pm 1.85$ & 0.585 \\
\hline COPD & $4(12.1)$ & $4(6.3)$ & 0.331 \\
\hline $\mathrm{CHF}$ & $2(6.1)$ & $9(14.3)$ & 0.220 \\
\hline Malignancy & $2(6.1)$ & $0(0.0)$ & 0.048 \\
\hline Endocrine & $8(24.2)$ & $17(27.0)$ & 0.771 \\
\hline Sepsis & $16(48.5)$ & $23(36.5)$ & 0.256 \\
\hline UTI & $4(12.1)$ & $6(9.5)$ & 0.692 \\
\hline \multicolumn{4}{|l|}{ Location of patient } \\
\hline Patients admitted to ICU & $9(27.3)$ & $20(31.7)$ & 0.650 \\
\hline Patients admitted to general wards & $24(72.7)$ & $43(68.3)$ & 0.650 \\
\hline \multicolumn{4}{|l|}{ Colistin treatment } \\
\hline Average colistin dose (mg/kg) & $2.83 \pm 0.50$ & $1.52 \pm 0.43$ & 0.001 \\
\hline Average duration of colistin treatment (days) & $12.76 \pm 13.38$ & $12.67 \pm 12.51$ & 0.974 \\
\hline Average length of stay (days) & $124.40 \pm 202.70$ & $113.10 \pm 340.80$ & 0.861 \\
\hline \multicolumn{4}{|l|}{ Concomitant nephrotoxic medications } \\
\hline Vancomycin & $18(54.6)$ & $46(73.0)$ & 0.068 \\
\hline Aminoglycosides & $5(15.2)$ & $4(6.3)$ & 0.160 \\
\hline NSAID & $11(33.3)$ & $29(46.0)$ & 0.231 \\
\hline ACEI/ARBS & $4(12.1)$ & $10(15.9)$ & 0.621 \\
\hline Furosemide & $15(45.5)$ & $36(57.1)$ & 0.276 \\
\hline \multicolumn{4}{|l|}{ Causative organisms } \\
\hline $\mathrm{AB}(\mathrm{n}=59)$ & $25(75.8)$ & $34(54.0)$ & 0.037 \\
\hline PA $(n=39)$ & $8(24.2)$ & $31(49.2)$ & 0.018 \\
\hline $\mathrm{KP}(\mathrm{n}=8)$ & $2(6.1)$ & $6(9.5)$ & 0.56 \\
\hline
\end{tabular}

Mean values $( \pm \mathrm{SD})$ and the number of cases with their relevant percentage in brackets

COPD, chronic obstructive pulmonary disease; CHF, congestive heart failure; UTI, urinary tract infection; ICU, intensive care unit; NSAID, non-steroidal anti-inflammatory drugs; ACEI, angiotensin converting enzyme inhibitor; ARBS, angiotensin II receptor blockers; AB, Acinetobacter baumannii; PA, Pseudomonas aeruginosa; KP, Klebsiella pneumoniae

\section{Efficacy and safety outcomes (Table 2)}

\section{Clinical cure analysis}

HDC was associated with a greater probability of clinical cure (30\%) compared to LDC (21\%), however the difference was not found to be significant $(p=0.292)$.

\section{Nephrotoxicity analysis}

Safety was measured as number of incidents of nephrotoxicity avoided by comparing treatment with HDC and LDC. There was a significant difference in the incidence of nephrotoxicity, $30 \%$ and $8 \%(p=0.004)$ between the HDC and LDC
Table 2 Outcomes

\begin{tabular}{llcc}
\hline & $\begin{array}{l}\text { High dose colistin } \\
(\mathrm{n}=33) \mathrm{n}(\%)\end{array}$ & $\begin{array}{l}\text { Low dose colistin } \\
(\mathrm{n}=63) \mathrm{n}(\%)\end{array}$ & $p$ value \\
\hline Clinical cure & $10(30)$ & $13(21)$ & 0.292 \\
Nephrotoxicity & $10(30)$ & $5(8)$ & 0.004 \\
\hline
\end{tabular}

groups, respectively. Using this data, we calculated the percentage of patients in which nephrotoxicity was avoided with HDC $(100-30=70 \%)$ and with LDC $(100-8=92 \%)$. 


\section{Multivariate analysis for dose by safety and efficacy measures $(n=96)$ (Table 3$)$}

Multivariate analysis revealed significant nephrotoxicity within high dose colistin arm ( $p$ value 0.011 ). Multivariate analysis further revealed that high dose colistin did not achieve better cure compared to low dose colistin (OR 1.74, 95\% CI $0.63-4.80 ; p=0.283)$. However, patients treated with HDC had a greater chance of developing nephrotoxicity (OR 4.71, 95\% CI 1.42-15.67; $p=0.011$ ).

\section{Costs}

Average total direct costs per episode of colistin treatment in the LDC and HDC groups were SAR 24,718.42 and SAR $27,775.25$, respectively. The main components of costs were days of ICU stay (18.8\% and $24.37 \%$ ) and physician visits (20.5\% and $18.37 \%$ ) for patients in LDC and HDC groups, respectively. Direct costs for the treatment duration in both arms listed in Table 4.

\section{Cost effectiveness}

Based on the efficacy and safety outcomes and the costs attached to each outcome, it was possible to calculate the

Table 3 Multivariate Analysis for dose by safety and efficacy measures $(\mathrm{n}=96)$

\begin{tabular}{llll}
\hline & OR & $95 \%$ CI & $p$ value \\
\hline Clinical cure & 1.74 & $0.63-4.80$ & 0.283 \\
Nephrotoxicity & 4.71 & $1.42-15.67$ & 0.011 \\
\hline
\end{tabular}

OR odds ratio
IC, CER and ICER. When LDC and HDC were compared using IC, LDC was the dominant strategy compared to HDC in terms of number of incidents of nephrotoxicity avoided (IC = SAR 3056.28 per nephrotoxicity avoided).

\section{Sensitivity analysis}

To reflect the uncertainty inherent in the analysis, a series of univariate (one-way) sensitivity analyses was performed for all parameters in the model to assess the effect of varying certain parameters by $25 \%$. Changes in the incremental costs are depicted in a tornado diagram (Fig. 2). Analyses were performed using Microsoft Excel (2003; Microsoft Corp, Redmond, WA, USA). The incremental costs per patient cured and incremental costs per nephrotoxicity avoided were most sensitive to the cost of physician visits, ICU days and general ward days. However, varying these parameters by $25 \%$ did not change the conclusions.

\section{Discussion}

Several studies have reported on the efficacy of LDC in the treatment of MDR GNB [7, 8, 11] and because of the financial burden that NP places on healthcare systems worldwide, we constructed an economic model to compare LDC and HDC

Our study design was similar to that used by Bounthavong et al. [20] in their cost effectiveness analysis of linezolid versus vancomycin in MRSA complicated skin and soft tissue infection. Whereas the authors of that study used past clinical trials to obtain the different probability parameters, our study utilized the probabilities from the retrospective analysis of patients treated at our institution during the study period. The outcomes of our study
Table 4 Mean direct cost of treatment per patient

\begin{tabular}{lccc}
\hline Daily costs & $\begin{array}{l}\text { Low dose colistin }(\mathrm{n}=63) \\
\text { mean } \pm \text { SD }\end{array}$ & $\begin{array}{l}\text { High dose colistin }(\mathrm{n}=33) \\
\text { mean } \pm \text { SD }\end{array}$ & $p$ value \\
\hline GW consultant visits & $1821.38 \pm 1788.57$ & $2069.54 \pm 1972.18$ & 0.535 \\
ICU consultant visits & $2421.05 \pm 4252.24$ & $3733.64 \pm 7804.30$ & 0.288 \\
GW days & $4738.10 \pm 6759.02$ & $4121.21 \pm 6412.91$ & 0.667 \\
Staff physician visits & $5066.67 \pm 5007.35$ & $5103.03 \pm 5354.47$ & 0.974 \\
ICU days & $4666.67 \pm 8939.76$ & $6772.73 \pm 14,344.04$ & 0.379 \\
Renal panel & $1013.33 \pm 1001.47$ & $1020.61 \pm 1070.89$ & 0.974 \\
GW nurse visits & $1266.67 \pm 1251.84$ & $1275.76 \pm 1338.62$ & 0.974 \\
ICU nurse visits & $933.33 \pm 1797.95$ & $1354.55 \pm 2868.81$ & 0.379 \\
Basic screen & $317.02 \pm 312.69$ & $318.94 \pm 334.65$ & 0.978 \\
CBC tests & $1013.33 \pm 1001.47$ & $1020.61 \pm 1070.89$ & 0.974 \\
Cost of colistin & $1129.91 \pm 847.51$ & $756.16 \pm 495.83$ & 0.022 \\
Laboratory cultures & $340.48 \pm 286.65$ & $228.48 \pm 189.56$ & 0.046 \\
Mean total costs & $27,775.24 \pm 31,893.63$ & $24,718.42 \pm 21,166.64$ & 0.576 \\
\hline
\end{tabular}


Fig. 2 Sensitivity analysistornado diagram. icu intensive care unit, $g w$ general ward, $c b c$ complete blood count

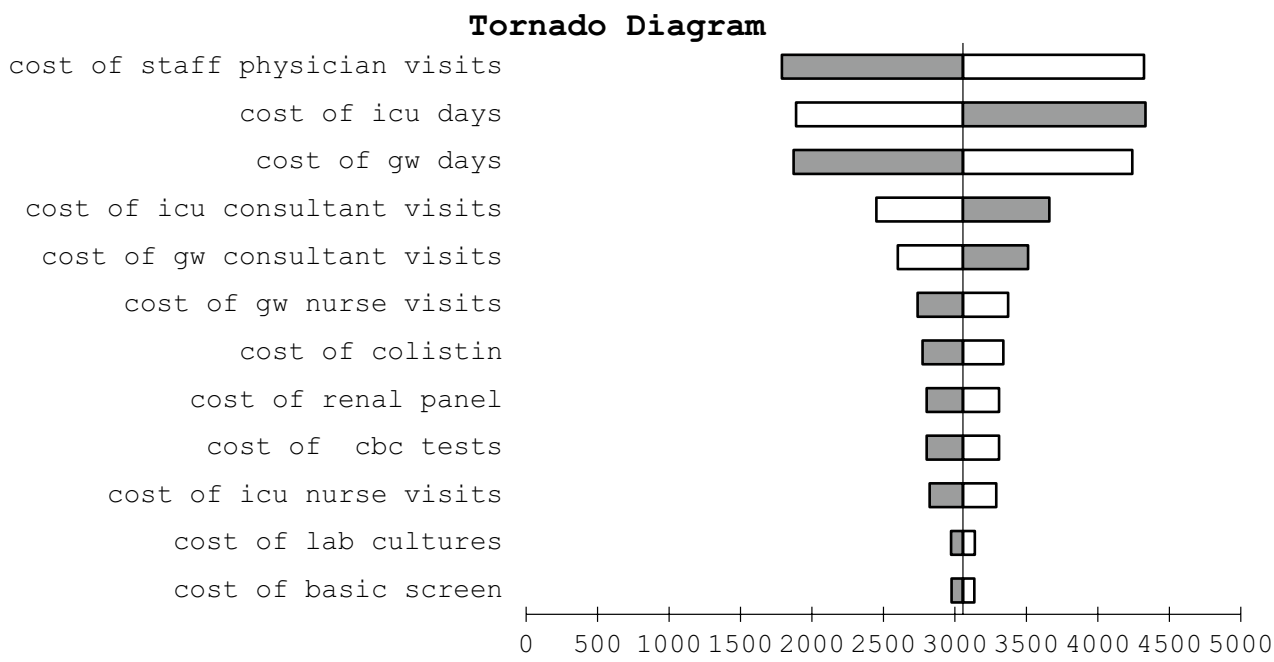

Abbreviation: $\mathrm{icu}=$ intensive care $\mathrm{unit} ; \mathrm{gw}=$ general ward; $\mathrm{cbc}=$ complete blood count were also calculated in the same manner as that utilized by Bounthavong et al.

A German cost effectiveness analysis of linezolid versus vancomycin in NP conducted by De Cock et al. [21] used a similar methodology to Bounthavong et al. The German authors, however, used a Delphi panel to supplement the clinical trial data as well as economic data when these were not available from the trials. Conditions of clinical trials are likely to be different from real life practice and our study used real life data.

The findings of this study show that there is no significant difference in clinical cure between HDC and LDC ( $30 \%$ vs. $21 \% ; p=0.292)$. The low clinical cure rates are similar to those of Kalin et al. [7] but much lower than those obtained by Yilmaz et al. [11].

Our findings also show that LDC produced significantly less nephrotoxicity than HDC ( $8 \%$ vs. $30 \%$; $p=0.004)$. The low incidence of nephrotoxicity with LDC is similar to that of Yilmaz et al. [11] who reported an incidence of $7.7 \%$ as well as those of Zaidi et al. [8] who reported a rate of $12.8 \%$ in patients on LDC.

Interpretation of the ICERs per additional patient cured and per nephrotoxicity avoided is appropriate considering that the costs and benefits relate to the model time horizon of colistin treatment. Using HDC, an investment of SAR 33,964.71 would be required per additional patient cured.

The use of LDC on the other hand would provide the same efficacy as HDC and realize a saving of SAR $13,894.66$ due to nephrotoxicity avoided.

\section{Limitations}

This was a retrospective single center study and this only provides insight into colistin use for NP at King Abdulaziz Hospital. A randomized controlled study would be preferred however limitations of resources did not warrant same. Due to the limited number of evaluable patients, the small sample size included in this study did not provide the required power. A larger sample would allow for more robust results.

Resistance to colistin is a major concern since this is frequently the only available medicine against MDR-GNB. Colistin resistance has been reported to be due to inadequate colistin dosing [22]. Higher doses seem to be more beneficial but no optimal dose has yet been defined. The effect of resistance was not investigated in our model and future studies should investigate whether resistance patterns would change the outcomes.

There is a possibility of selection bias since patients in the low dose group were of higher weight compared to the high dose group and it is possible that the physicians prescribed lower doses of colistin for these patients.

This study defined nephrotoxicity using change in serum creatinine, as this was the most readily available in patient's charts. The use of RIFLE criteria would have been ideal as this is now recognized as the standard diagnostic criteria [19].

Antibiotics used to treat other gram-negative and grampositive organisms were assumed to be equivalent between both groups and thus not included into the model. Inclusion of other treatment costs could affect the results. 


\section{Conclusion}

This study provides a deeper insight into the use of colistin in NP despite the assumptions and limitations. Based on the results of this study, KAH should adopt the LDC strategy for treatment of NP caused by MDR-GNB while taking cognizance of local resistance patterns. Further studies are needed to investigate the true impact of using LDC on healthcare costs.

Acknowledgements We thank Ms. Analyn Crisostomo (B.Pharm) for collecting data on some study variables and Ms. Iman Moustafa (M.Pharm) for assistance with layout and proof reading.

Funding None.

Conflicts of interest The authors declare that they have no conflicts of interest.

\section{References}

1. Focaccia R, Gomes Da Conceicao OJ. Pneumonia hospitalar. Rev Bras Med. 1994;51(SPEC. ISS.):95-8.

2. Nair GB, Niederman MS. Nosocomial pneumonia. Lessons learned. Crit Care Clin. 2013;29(3):521-46.

3. Aly M, Balkhy HH. The prevalence of antimicrobial resistance in clinical isolates from Gulf Corporation Council countries. Antimicrob Resist Infect Control. 2012;1(1):26.

4. Tao L, Hu B, Rosenthal VD, Gao X, He L. Device-associated infection rates in 398 intensive care units in Shanghai, China: International Nosocomial Infection Control Consortium (INICC) findings. Int J Infect Dis. 2011;15(11):e774.

5. Navoa-Ng JA, Berba R, Galapia YA, Rosenthal VD, Villanueva VD, Tolentino MCV, et al. Device-associated infections rates in adult, pediatric, and neonatal intensive care units of hospitals in the Philippines: International Nosocomial Infection Control Consortium (INICC) findings. Am J Infect Control. 2011;39(7):548-54.

6. Scott II RD. The direct medical costs of healthcare-associated infections in U.S. hospitals and the benefits of prevention. CDC; 2009.

7. Kalin G, Alp E, Coskun R, Demiraslan H, Gündogan K, Doganay $\mathrm{M}$. Use of high-dose IV and aerosolized colistin for the treatment of multidrug-resistant Acinetobacter baumannii ventilatorassociated pneumonia: do we really need this treatment? J Infect Chemother. 2012;18(6):872-7.

8. Zaidi STR, Al Omran S, Al Aithan ASM, Al Sultan M. Efficacy and safety of low-dose colistin in the treatment for infections caused by multidrug-resistant gram-negative bacteria. J Clin Pharm Ther. 2014;39(3):272-6.
9. Pintado V, San Miguel LG, Grill F, Mejía B, Cobo J, Fortún J, et al. Intravenous colistin sulphomethate sodium for therapy of infections due to multidrug-resistant gram-negative bacteria. J Infect. 2008;56(3):185-90.

10. Montero M, Horcajada JP, Sorlí L, Alvarez-Lerma F, Grau S, Riu $\mathrm{M}$, et al. Effectiveness and safety of colistin for the treatment of multidrug-resistant Pseudomonas aeruginosa infections. Infection. 2009;37(5):461-5.

11. Yilmaz GR, Baştuğ AT, But A, Yildiz S, Yetkin MA, Kanyilmaz $\mathrm{D}$, et al. Clinical and microbiological efficacy and toxicity of colistin in patients infected with multidrug-resistant gram-negative pathogens. J Infect Chemother. 2013;19(1):57-62.

12. Dubrovskaya Y, Chen T-Y, Scipione MR, Esaian D, Phillips MS, Papadopoulos J, et al. Risk factors for treatment failure of polymyxin B monotherapy for carbapenem-resistant Klebsiella pneumoniae infections. Antimicrob Agents Chemother. 2013;57(11):5394-7.

13. Doshi NM, Mount KL, Murphy CV. Nephrotoxicity associated with intravenous colistin in critically ill patients. Pharmacotherapy. 2011;31(12):1257-64.

14. Falagas ME, Fragoulis KN, Kasiakou SK, Sermaidis GJ, Michalopoulos A. Nephrotoxicity of intravenous colistin: a prospective evaluation. Int J Antimicrob Agents. 2005;26(6):504-7.

15. Gauthier TP, Wolowich WR, Reddy A, Cano E, Abbo L, Smith LB. Incidence and predictors of nephrotoxicity associated with intravenous colistin in overweight and obese patients. Antimicrob Agents Chemother. 2012;56(5):2392-6.

16. Pogue JM, Lee J, Marchaim D, Yee V, Zhao JJ, Chopra T, et al. Incidence of and risk factors for colistin-associated nephrotoxicity in a large academic health system. Clin Infect Dis. 2011;53(9):879-84.

17. Chan JD, Graves JA, Dellit TH. Antimicrobial treatment and clinical outcomes of carbapenem-resistant Acinetobacter baumannii ventilator-associated pneumonia. J Intensive Care Med. 2010;25(6):343-8.

18. Magiorakos A, Srinivasan A, Carey RB, Carmeli Y, Falagas ME, Giske CG, et al. bacteria: an international expert proposal for interim standard definitions for acquired resistance. Clin Microbiol Infect. 2011;18:268-81.

19. Kellum JA, Lameire N, Aspelin P, Barsoum RS, Burdmann EA, Goldstein SL, et al. KDIGO clinical practice guideline for acute kidney injury. Kidney Int Suppl. 2012;2(1):1-138.

20. Bounthavong M, Hsu DI, Okamoto MP. Cost-effectiveness analysis of linezolid vs. vancomycin in treating methicillinresistant Staphylococcus aureus complicated skin and soft tissue infections using a decision analytic model. Int J Clin Pract. 2009;63(3):376-86.

21. De Cock E, Krueger WA, Sorensen S, Baker T, Hardewig J, Duttagupta S, et al. Cost-effectiveness of linezolid vs vancomycin in suspected methicillin-resistant staphylococcus aureus nosocomial pneumonia in Germany. Infection. 2009;37(2):123-32.

22. Michalopoulos AS, Falagas ME. Colistin: recent data on pharmacodynamics properties and clinical efficacy in critically ill patients. Ann Intensive Care. 2011;1(1):30. 